\title{
Comparison of some EMD based Technique for Baseline Wander Correction in Fetal ECG Signal
}

\author{
P K Ghosh \\ Department of ECE \\ College of Engineering \& Technology, Mody \\ University \\ Lakshmangarh, Sikar-332311, Rajasthan, India
}

\author{
Deepika Poonia \\ College of Engineering \& Technology, Mody \\ University \\ Lakshmangarh, Sikar-332311, Rajasthan, India
}

\begin{abstract}
Baseline wander (BW) is a low frequency artifact in biomedical electronic recordings. It is usually caused by patient's respiration or movement of equipments. The removal of this artifact is important in ECG recordings for reliable visual interpretation. This paper presents the implementation of Empirical Mode Decomposition (EMD), Ensemble Empirical Mode Decomposition (EEMD) and EMD based method to remove this disturbance. The EMD based technique serves as an efficient method to remove baseline wander with minimum signal distortion. The results highlights the main differences among all different methods and also show that the EMD based technique is able to remove best baseline wander.
\end{abstract}

\section{Keywords}

Baseline wander, Empirical Mode Decomposition (EMD), Ensemble Empirical Mode Decomposition (EEMD)

\section{INTRODUCTION}

THE ECG signal is a representation of the bio electrical activity of the heart, representing the cyclic contraction and relaxations of the human heart muscles. ECG analysis is widely used as a reliable technique for the diagnosis of the cardiac failure and cardio vascular disease [1]. To acquire the electrocardiogram signal, ECG devices with number of electrodes (3-12) can be used. Multi lead systems are also available that having 12 and up to 120 electrodes are also available [2]. ECG recordings obtained by placing electrodes on the surface of subject's body are inevitably contaminated by several different types of artifact.

The transmission of ECG signal often introduces noise because of poor channel conditions and there are other types of noise inherent in the data collection process. The main sources of such disturbances are - (1) The wideband noise (baseline wander) is mainly caused by breathing or movements and (2) the narrow-band noise such as power line interference or mechanical forces which are acting on the electrodes is caused by the muscle activity. Both type of substantial disturbances affected by the motion of the patient or the ECG leads [3]. Base line wander (or trend) lies between 0.15 and $0.3 \mathrm{~Hz}$. The power line interference is centered at 60 $\mathrm{Hz}$ (or $50 \mathrm{HZ}$ ) with a bandwidth of less than $1 \mathrm{~Hz}$ [2]. The separation of the ECG signal from these disturbances allows proper analysis and display.

Many researchers have been reported in the literature for removal of baseline wander noise. Some relevant contributions have proposed using some different techniques, such as the adaptive filtering [4], wavelet transform [5-6], EMD [7] and EEMD [8].

The aim of this paper is to compare three different existing techniques of base line wander removal. The EMD algorithm was recently introduced in [7] a technique for obtaining oscillatory modes present in the data. The EMD allows overcoming the intrinsic limitations of wavelet analysis. The EMD is a good tool for artifact reductions applications. This motivates the use of EMD in ECG enhancement. In this work we address a comparison among EMD algorithm, EEMD algorithm and EMD based method.

This paper is organized as follows: Section II details the mathematical basis of EMD algorithm, EEMD algorithm and EMD based method for baseline removal. The simulation results and comparison with published results are highlighted in section III. Conclusions are drawn in Section IV.

\section{MATHEMATICAL BASIS}

In this section the mathematical background of the EMD algorithm, EEMD algorithm and EMD based method for baseline removal is summarized.

\subsection{EMD Algorithm}

The empirical mode decomposition is an adaptive and fully data-driven technique that does not require any priori. The EMD has been introduced by Huang et.al [7] to non-linear and non-stationary time series such as bio-medical signals. EMD attempts to decompose a time series into intrinsic oscillations by exploiting both local temporal and structural characteristics of the data. The starting point of EMD is to estimate a signal as a sum of low frequency and the detail components for high frequencies components are referred to as Intrinsic Mode Functions (IMFs) and the low frequency components are called residuals. The process of finding the IMF is called the shifting process [9], is described as follows

(a) Firstly we have to identify all the local maxima and minima. Generate a upper envelope $e_{u p}(t)$ as a cubic spline curve by connecting all the local maxima. Similarly, generate lower envelope $e_{\text {low }}(t)$ as a spline curve by connecting all the local minima.

(b) The local mean of these envelops is determined and denoted as

$$
m_{10}(t)=\left(e_{u p}(t)+e_{l o w}(t)\right) / 2
$$

(c) This local mean is subtracted from the signal $x(t)$ and the first proto-IMF $p_{10}(t)$ is obtained as

$$
p_{01}=x(t)-m_{01(t)}
$$

(d) Repeat the above procedure to get

$$
p_{11}(t)=p_{01}(t)-m_{11}(t)
$$


Repeat $\quad p_{1 k}(t)=p_{1(k-1)}(t)-m_{1 k}(t)$ until the proto-IMF $p_{1 k}(t)$ is an IMF. Here $m_{1 k}(t)$ is the local mean of $p_{1(k-1)}(t)$.

The shifting process is applied repetitively to the proto-IMF $p_{1 k}(t)$ until the first IMF $a_{1}(t)$ is obtained. To terminate the shifting process a stopping criterion is the sum of difference (SD) [3]:

$$
S D=\sum_{t=0}^{T} \frac{\left|p_{1(k-1)}(t)-p_{1 k}(t)\right|^{2}}{p_{1(k-1)}^{2}(t)}
$$

The first IMF $a_{1}(t)$ is obtained when the SD is smaller than a threshold; this first component contains the finest temporal scale in the signal. To obtain a series of IMFs, the procedure is described as follows

(1) The residue $r_{1}(t)$ is obtained by subtracting the first IMF $a_{1}(t)$ from signal $x(t)$ [9]. It is written as

$$
r_{1}(t)=x(t)-a_{1}(t)
$$

(2) Note that the residue $r_{1}(t)$ still contains some useful information of longer period components. Therefore, this residue is treated as a new signal. Apply the above shifting process on the entire residue $r_{j}(t)$ :

$$
r_{1}(t)-a_{2}(t)=r_{2}(t), \ldots ., r_{n-1}(t)-a_{n}(t)=r_{n}(t)
$$

(3) The whole repetition process terminates when the residue, $r_{n}(t)$ is a constant, a monotonic slope, or a function with only one extremum.

The EMD of the original signal can be reconstructed by combining equations (3) and (4)

$$
x(t)=\sum_{i=1}^{n} a_{i}(t)+r_{n}(t)
$$

For convenience, we refer to $a_{i}(t)$ as the $i^{\text {th }}$ order IMF.

The last residue $r_{n}(t)$ is usually considered as a last IMF $a_{n+1}(t)$ and equation (5) is rewritten as

$$
x(t)=\sum_{i=1}^{n+1} a_{i}(t)
$$

The higher order IMF contains fewer signal components while the lower order IMF contains more signal components. The result of the EMD produces $n$ IMFs and a residue signal. The flowchart of this algorithm is depicted in figure 1 .

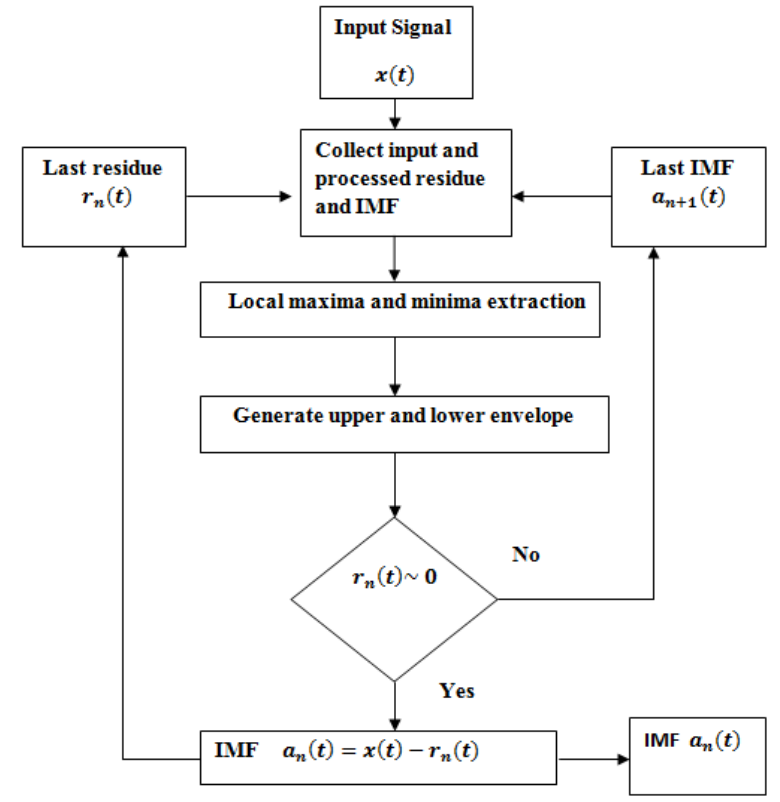

Fig 1: Flow Chart of EMD algorithm

\subsection{EEMD Algorithm}

The EMD algorithm is very sensitive to noise in ECG signal. Due to mode mixing, this algorithm can lead complications. The mode mixing effect is defined as an IMF that includes oscillations of a component of similar scale assigned to different IMFs. It can also be due to the presence of a transient spectral component in the ECG signal. An extension to the EMD algorithm was presented in [8], is called Ensemble Empirical Mode Decomposition (EEMD), which removes this mode mixing effect. The EEMD algorithm uses an average number of ensembles of the EMD algorithm as the optimum choice of IMFs.

The main objective of the EEMD algorithm is to add white noise into the signal with many numbers of trials. Each trial has different noise, and the added noise can be canceled out on average, if the number of trials is sufficient. Hence, as more and more trials are added to the ensemble, the residual part is the signal. The process of EEMD method is as follows [8]:

(i) White noise series $w(t)$ is added to signal $x(t)$ in following manner

$$
x_{1}(t)=x(t)+w(t)
$$

(ii) Signal $x_{1}(t)$ decomposes by using EMD algorithm.

(iii) Step (i) and step (ii) are repeated until the trial numbers, each time with different added white noise series of the same power at each time. $b_{i j}(t)$ is achieved as the new IMF combination, where $i$ the iteration number and $j$ is the scale of IMF.

(iv) Estimate the mean of the final IMF of the decompositions as

$$
b_{j}(t)=\sum_{i=1}^{n} b_{i j}(t)
$$

where $n$ denotes total number of trials. 


\subsection{EMD based method}

This EMD based method is proposed in [3]. BW corresponds to a low frequency of the signal, however it can be considered as the summation of certain higher order IMFs. In fact, fewer ECG signal components and major BW components are located in higher IMFs while more signal components and fewer BW components are located in lower IMFs. BW may have multiple extrema and zero crossing which violets the residue definition. Therefore, the residue, which can also regarded as the last IMF, may not correspond to baseline wander.

The baseline wander must be separated from the last IMFs. The number of IMFs that contribute to the BW is referred as BW order. BW is estimated here via a "multiband" filtering approach. The estimated BW is then subtracted from the signal. A bank of low pass filters is applied to the last IMFs. The baseline wander is estimated by the sum of the outputs of this filter bank.

By performing the EMD on signal $x(t)$, we obtain all the IMFs, which taken from equation (6). The BW is denoted as $N$; a bank of low pass filters $d_{i}(t), i=1,2, \ldots \ldots . . N$, is assigned and this filter bank is applied to the IMFs starting from the last $a_{n+1}(t)$. The outputs of the filter bank are

$$
\begin{aligned}
& h_{1}(t)=d_{1}(t) * a_{n+1}(t) \\
& h_{2}(t)=d_{2}(t) * a_{n}(t) \\
& \cdot \\
& \cdot \\
& h_{N}(t)=d_{N}(t) * a_{n-N+2}(t)
\end{aligned}
$$

where $*$ denotes the convolution. The cut off frequency of the first low pass filter $d_{1}(t)$ is set to be $\omega_{0}$ and the cutoff frequency of the $k^{\text {th }}$ filter [3] is set as

$$
\omega_{k}=\frac{\omega_{0}}{M^{k-1}}
$$

where $M>1$ is a frequency folding number. The outputs $h_{i}(t)$ extract the BW component in each IMF. Therefore, this output can be used to determine the $\mathrm{BW} \operatorname{order} N$. The variance of each $h_{i}(t)$ is determined [3] as:

$$
\operatorname{var}\left\{h_{i}(t)\right\}=\frac{1}{L-1} \sum_{t=0}^{L-1}\left[h_{i}(t)-\mu_{h_{i}}\right]^{2}
$$

Where $\mu_{h_{i}}$ is mean value of the output $h_{i}(t)$. We choose $N$ such that $\operatorname{var}\left\{h_{N+1}(t)\right\}<\xi$ and $\operatorname{var}\left\{h_{N}(t)\right\} \geq \xi$, starting from the last IMF. Where $\xi$ is an appropriate established threshold. The parameter $\omega_{0}, M, \xi$ can be selected according to the baseline wander behavior [3].
After the determination of BW order $N$, the output of all the filter are synthesized to form the estimate as

$$
\hat{h}(t)=\sum_{i=1}^{N} h_{i}(t)
$$

The reconstructed signal is obtained by subtracting the estimated $\hat{h}(t)$ from the signal $x(t)$. Therefore, the reconstructed signal after BW removal is

$$
\tilde{x}(t)=x(t)-\hat{h}(t)
$$

\section{SIMULATION RESULTS AND DISCUSSIONS}

The experiments are performed on the database of Non Invasive Fetal ECG database [10], having baseline wander noise shown in figure 2 .

Firstly the EMD algorithm is applied on this database, and then produced 10 IMFs (in form of higher to lower order) are shown in figure 3 . The signal is reconstructed by subtracting $7^{\text {th }}$ IMF from original database. The reconstructed ECG signal by using EMD method is shown in figure 4 .

An extension to the EMD method that is, the EEMD method is applied to the database which is shown in figure 2 . The 10 IMFs which are produced by this method are shown in figure 5. After applying EEMD method, the signal can be reconstructed by subtracting $8^{\text {th }}$ IMF from original database which is shown in figure 6 .

Baseline wander are located in higher order IMFs (figure 3) and the baseline wander spread over the last IMFs. A bank of low pass filters are applied to the last IMFs. The sum of output of this filter bank serves as estimated baseline wander. The reconstructed signal is obtained by removing this estimated baseline wander from the original signal, which is described in section II (part C) is shown in figure 7.

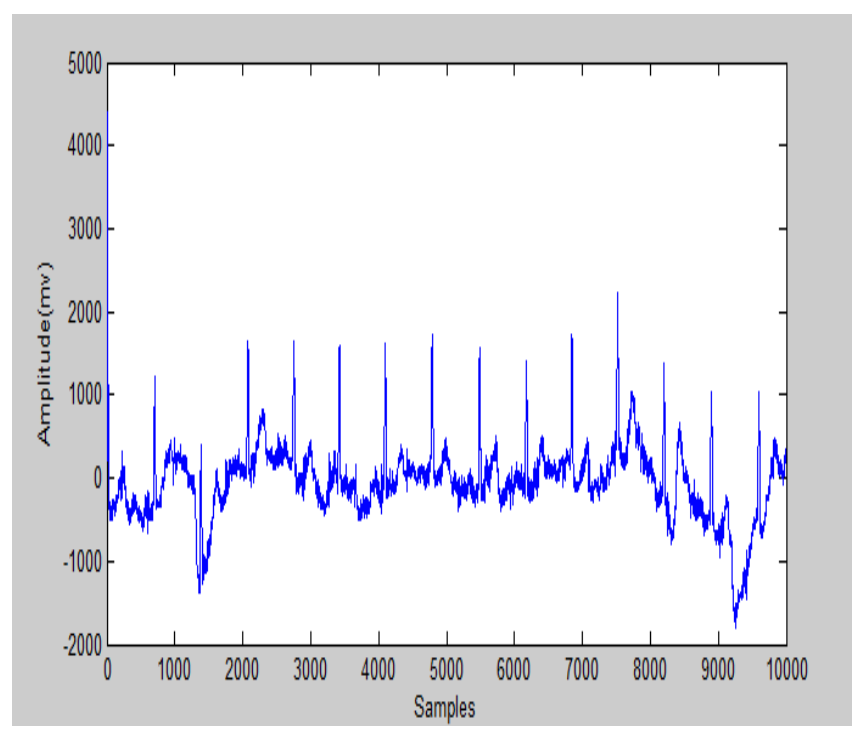

Fig 2: Non Invasive Fetal ECG database 


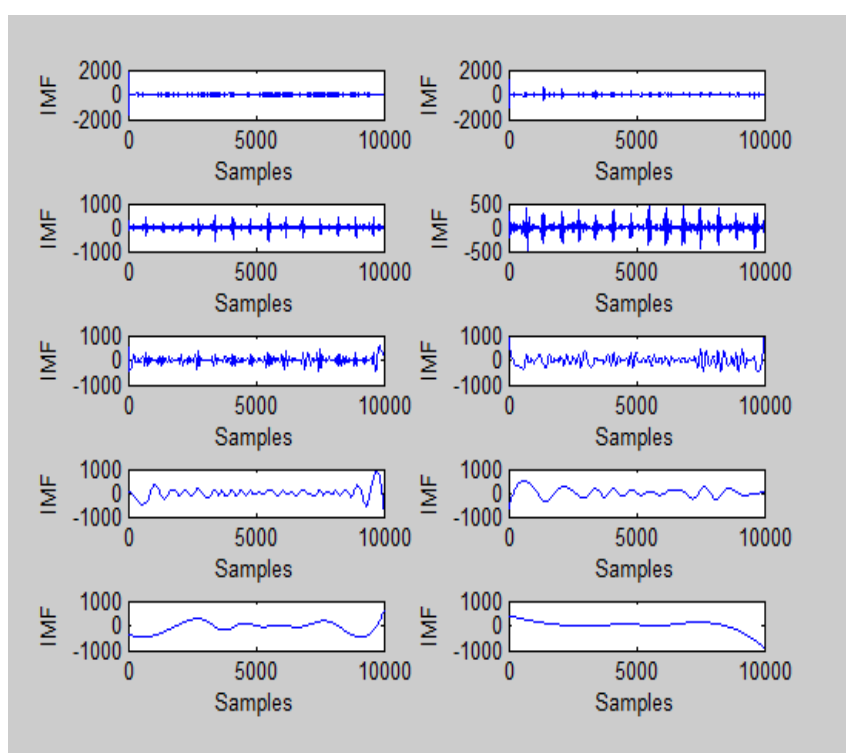

Fig 3: IMFs (1-10) obtained by EMD algorithm

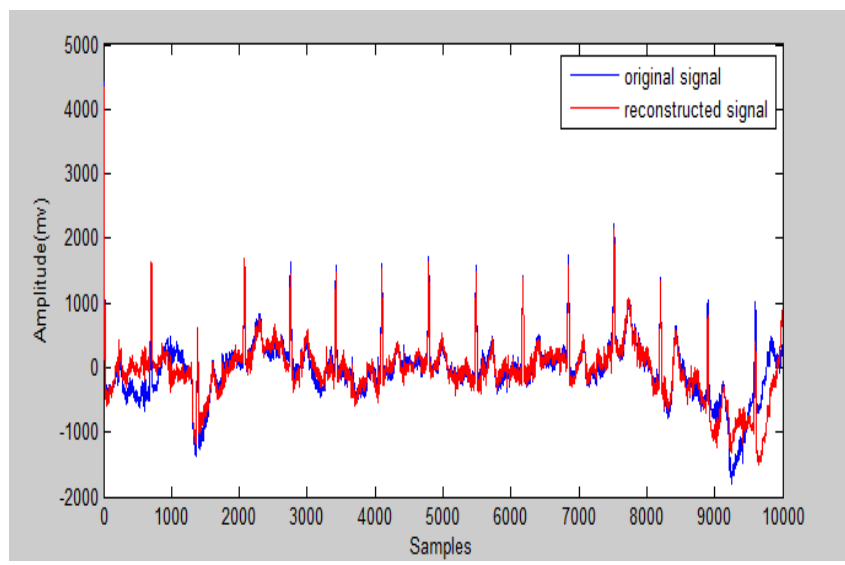

Fig 4: Reconstructed signal from EMD algorithm

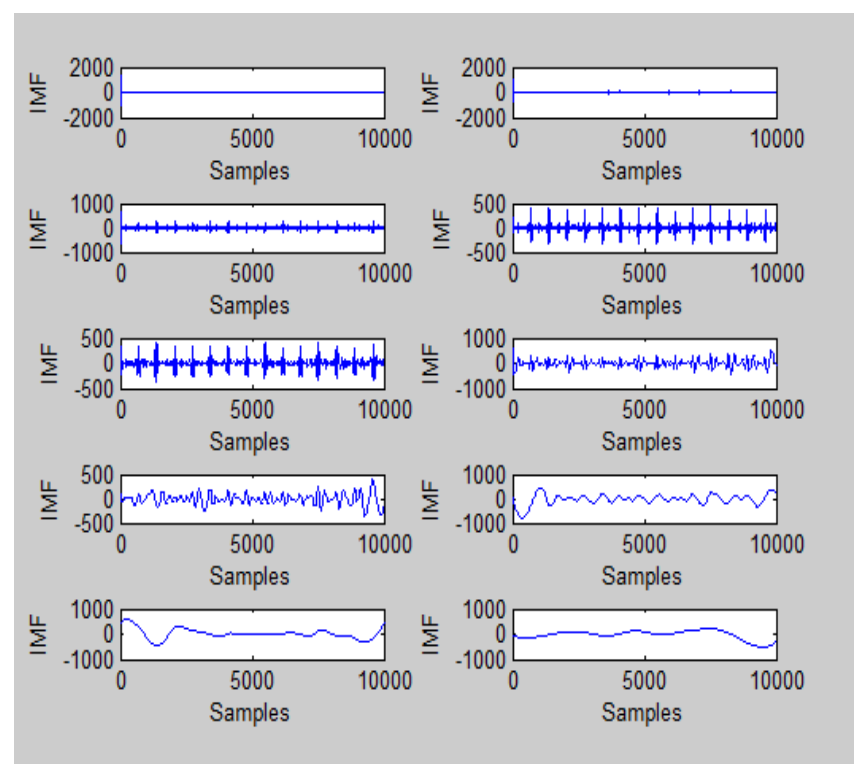

Fig 5: IMFs (1-10) obtained by EEMD algorithm

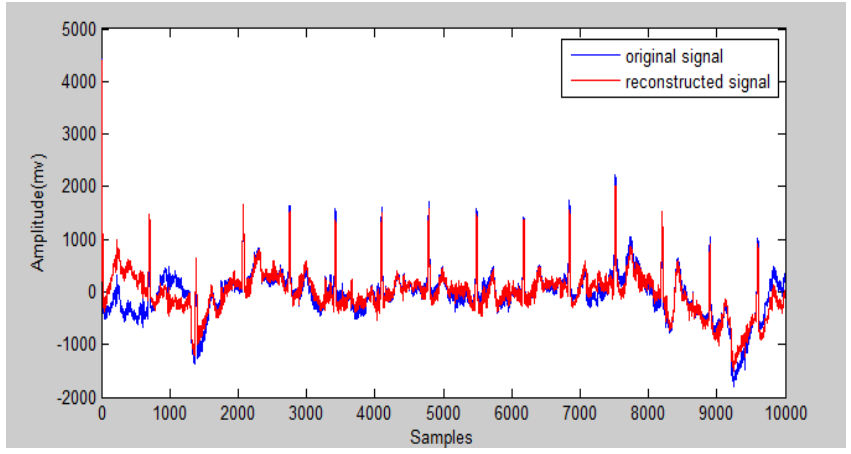

Fig 6: Reconstructed signal from EEMD algorithm

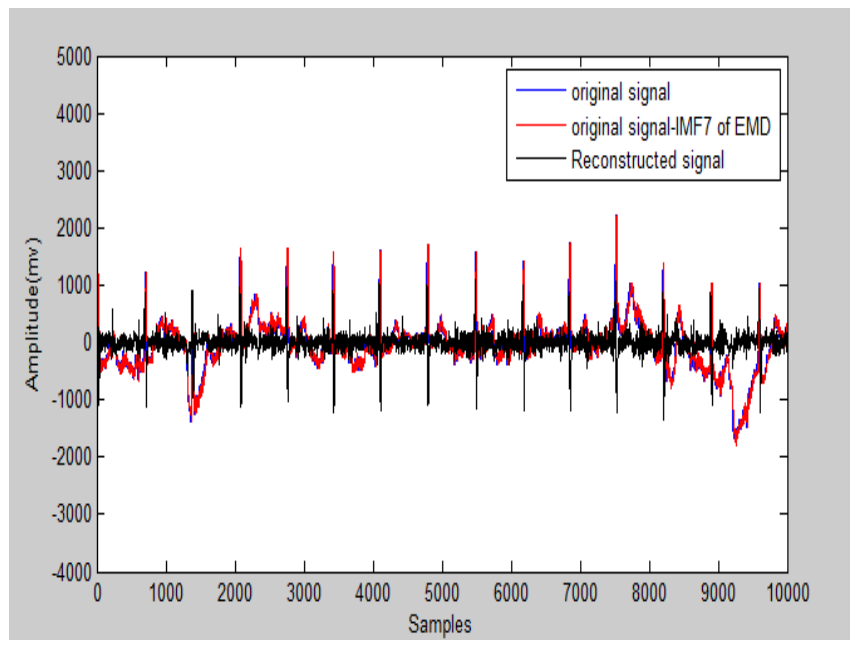

Fig 7: Reconstructed signal from EMD based method

The IMFs are produced by EMD algorithm and EEMD algorithm, shown in figure 3 and figure 5 respectively. Comparing the IMF component of the same level, EEMD has more concentrated and band limited components. We can see that baseline wander is in $7^{\text {th }}$ level in EMD and $8^{\text {th }}$ level in EEMD. ECG components are located between $3^{\text {rd }}$ to $6^{\text {th }}$ level in EMD and $3^{r d}$ to $7^{\text {th }}$ level in EEMD.

The reconstructed signals are shown in figure 4, figure 6 and figure 7 . The comparison between these signals show that the baseline removal using EMD method achieves best baseline wander removal. The cutoff frequency of high pass filter is experimentally $\omega_{0}$ set to $0.09 \mathrm{~Hz}, M$ is set to 20 and $\xi$ is set to 10 , which is described in section II (part C).

\section{CONCLUSIONS}

In this paper a comparison among three methods for baseline removal has been carried out. It has been shown how all techniques used are able to remove baseline wander noise from an ECG database. In particular, the EMD based method with high pass filtering leads to better results especially regard to the baseline wander noise removal.

Furthermore, by comparing the IMFs obtained by the EMD algorithm and EEMD algorithm, EEMD significantly remove the mode mixing effect. By comparing the IMF component of same level, EEMD has more concentrated and band limited components. Moreover, the EMD based technique which is used here can be applied in practical stress ECG tests and long term holder monitoring. 


\section{REFERENCES}

[1] Adam Gacek and Witold Pedrycz, ECG Signal Processing, Classification and Interpretation. SpringerVerlag London Limited, 2012.

[2] Miad Faezipour, Adnan Saeed, Chandrika Bulusu, Hlaing Minn and Lakshman Tamil, "A patient-adaptive profiling scheme for ECG beat classification," IEEE Trans. Inf. Technol. Biomed., vol. 14, no. 5, Sept. 2010.

[3] Manuel Blanco-Velasco, Binvei Weng and Kenneth E. Barner,"ECG signal denoising and baseline wander correction based on the empirical mode decomposion," ELSEVIER Comput. Biol. Med.38, pp. 1-13, 2008.

[4] V. Almenar and A. Albiol, "A new adaptive scheme for ECG enhancement," Signal Process.75(3), pp. 253-263, 1999.

[5] P. E. Tikkanen, "Non-linear wavelet and wavelet packet denoising of ECG signal," Biol. Cybern. 80(4), pp. 259267, 1999.

[6] Domenico Labate, Fabio La Foresta, Gianluigi Occhiuto, Francesco Carlo Morabito, Aime Lay-Ekuakille and Patrizia Vergallo, "Empirical mode decomposition vs. wavelet decomposition for the extraction of respiratory signal from singal-channel ECG: a comparison," IEEE Sensors Journal, vol. 13, no. 7, Jul. 2013.

[7] N. E. Huang, Z. Shen, S. R. Long, M. C. Wu, H. H. Shih, Q. Zheng, N.-C. Yen, C. C. Tung, and H. H. Liu, "The empirical mode decomposition and Hilbert spectrum for non-linear and non-stationary time series analysis," Proc. Royal Soc. London A, vol. 454, pp. 903-995, Mar. 1998.

[8] Zhaohua $\mathrm{Wu}$ and Norden E. Huang, "Ensemble empirical mode decomposition: a noise-assisted data analysis method," Advances in Adaptive Data Analysis $1(1)$, pp. 1-41, 2009.

[9] T. Y. Ji, Z. Lu, Q. H. Wu and Z. Ji, "Baseline normalization of ECG signals using empirical mode decomposition and Mathematical morphology, ELECTRONICS LETTERS, vol. 44, no. 2, Jan. 2008.

[10] physionet.org/cgi-bin/atm/ATM

\section{AUTHOR's PROFILE}

Dr. P. K. Ghosh was born in Kolkata, India in 1964 . He received his B.Sc (Hons in Physics), B.Tech and M.Tech degrees in 1986, 1989, and 1991, respectively from Calcutta University. He earned Ph.D.(Tech) degree in Radio Physics and Electronics in 1997 from the same University. He served National Institute of Science and Technology (Orissa), St. Xavier's College (Kolkata), Murshidabad College of Engineering and Technology (West Bengal), R. D. Engineering College (Uttar Pradesh) and Kalyani Government Engineering College (West Bengal) before he joins Mody University (Rajasthan). To his credit, he has more than 60 research papers in Journals of repute and conference proceedings. He is a life member of Indian Society for Technical Education (ISTE), New Delhi. His research interests are in the areas of wireless communications, digital signal processing, VLSI circuits \& devices.

Deepika Poonia was born in Rajasthan, India in 1993. She has done her B.Tech in Electronics and Communication from Rajasthan Technical University, Kota, Rajasthan. At present she is pursuing M.Tech in Signal Processing from Mody University. Her research interests are in the field of Biomedical Signal Processing and Digital Signal Processing. 